\title{
Aneurisma disecante de la arteria cerebelosa posteroinferior
}

\author{
J.J. Rivas; J. Domínguez; P. Bravo; J. Pérez y A.P. Avila
}

Servicio de Neurocirugía. Hospital Nuestra Señora de la Candelaria. Santa Cruz de Tenerife.

\section{Resumen}

Los aneurismas disecantes que afectan de forma exclusiva a la arteria cerebelosa posteroinferior (PICA) son muy raros. Su manifestación clínica dependerá del lugar de la pared arterial en que se produzca la disección, produciendo hemorragia subaracnoidea (HSA) o bien oclusión del vaso e isquemia. El diagnóstico del aneurisma se realiza en el estudio angiográfico mostrándose como una dilatación fusiforme de la arteria o como grados variables de estrechamiento y dilatación del vaso. La resonancia magnética $(\mathrm{RM})$ puede ser de gran ayuda detectando el hematoma intramural.

Presentamos un enfermo de 47 años que sufrió una HSA que toleró bien. El estudio angiográfico mostró una dilatación fusiforme de la porción anterobulbar de la PICA. El paciente fue intervenido realizándose una exclusión del segmento de la PICA afecto, colocando un clip proximal y otro distal al aneurisma. El postoperatorio transcurrió sin incidencias a pesar de presentar en la angiografía de control un vasoespasmo muy notable de todo el territorio vertebrobasilar.

Los aneurismas disecantes de la PICA y fundamentalmente los que se presentan con HSA deben se tratados a fin de prevenir el resangrado. Tanto los procedimientos quirúrgicos como los endovasculares se han revelado eficaces y con buenos resultados.

PALABRAS CLAVE. Aneurisma disecante. Arteria cerebelosa posteroinferior. Hemorragia subaracnoidea. Tratamiento endovascular. Tratamiento quirúrgico.

Dissecting aneurysm of the posterior cerebellar artery

\section{Summary}

Dissecting aneurysms affecting exclusively to the posterior inferior cerebellar artery (PICA) are rare entities. Depending on the dissecting plane of the

Recibido:10-06-06.Aceptado: 10-07-06 arterial wall, the clinical manifestations are subarachnoid hemorrhage (SAH) due to rupture or ischemia caused by stenosis or occlusion. Angiographic findings are fusiform dilatation with a narrowing of various degrees proximal to and distal to the fusiform lesion. Magnetic resonance imaging (MRI) can be useful demonstrating the intramural hematoma.

We report a 47-year-old man who suffered from SAH. He was neurologicaly intact and vertebral angiography demonstrated and fusiform aneurysm at the origin of the left PICA.

He was operated by trapping of the dissecting segment. The patient's postoperative course was uneventful despite of severe vasospasm showed in follow up angiography.

Aggressive treatment has been recommended for dissecting aneurysms of the PICA and specially for those presenting with SAH. Both the surgical and endovascular procedures are effective and with good results.

KEY WORDS. Dissecting aneurysm. Posterior inferior cerebellar artery. Subarachnoid hemorrhage. Endovascular therapy. Aneurysm surgery.

\section{Introducción}

Los aneurismas de la arteria cerebelosa posteroinferior (PICA) representan el 0,5-3\% de todos los aneurismas intracraneales ${ }^{9,16}$. Los de carácter disecante y espontáneos que afectan exclusivamente a la PICA son excepcionales y su verdadera incidencia es desconocida. Se han publicado 35 casos hasta la actualidad ${ }^{1,4,6,8,10,11,13-15,17,22,24,26,28-33,35}$. Presentamos el caso de un paciente que sufrió una hemorragia

Abreviaturas: AICA: antero inferior cerebellar artery; arteria cerebelosa antero inferior. HSA: hemorragia subaracnoidea. MRI: magnetic resonance imaging. PICA: postero inferior cerebellar artery; arteria cerebelosa postero inferior. RM: resonancia magnética. SAH: subarachnoid hemorrhage. TC: tomografia computarizada 

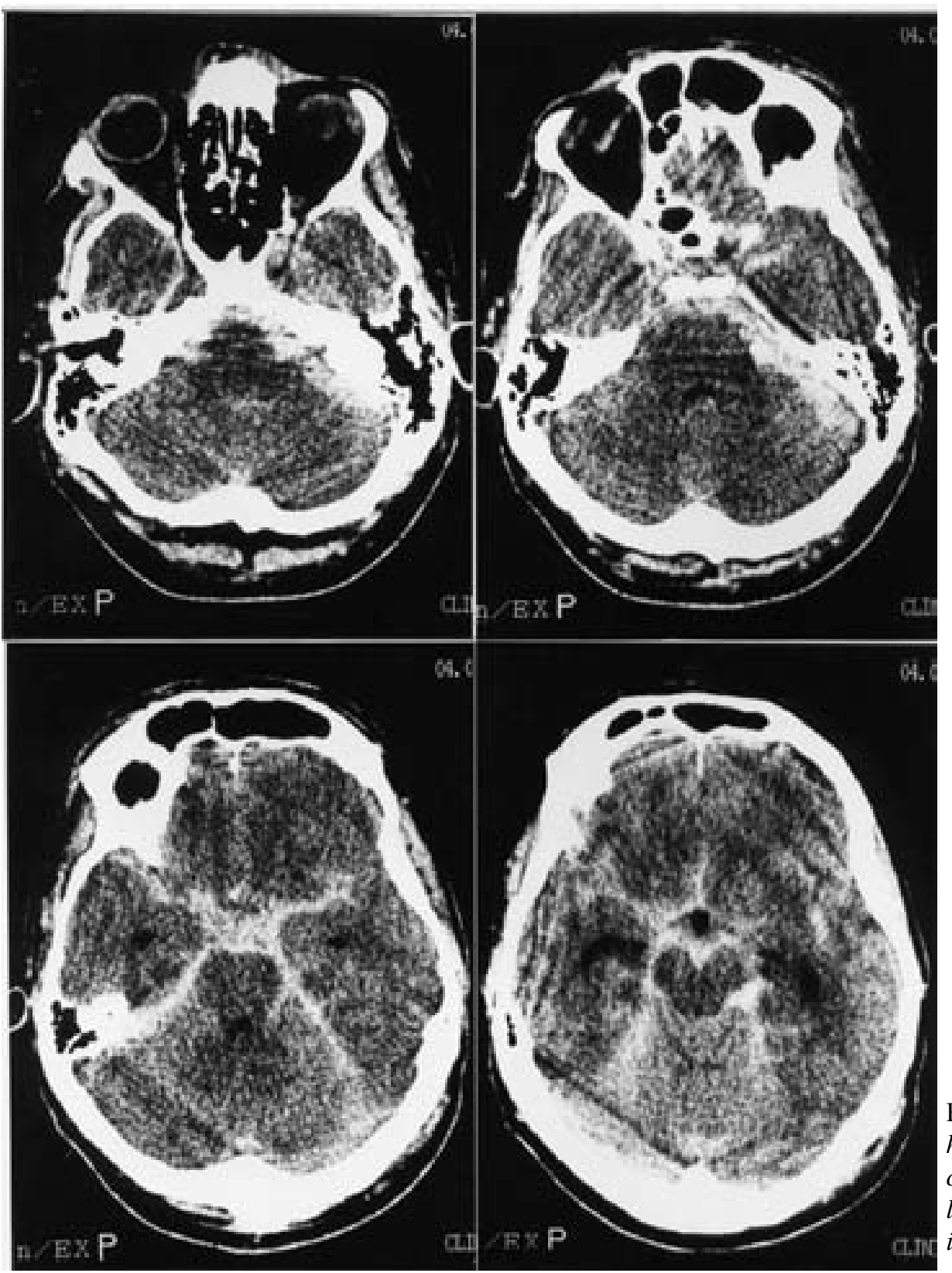

Figura 1. TC cerebral. Se aprecia hemorragia subaracnoidea en las cisternas de la base, peritruncales y del ángulo pontocerebeloso izquierdo.

subaracnoidea (HSA) a causa de la ruptura de un aneurisma disecante en la PICA izquierda. Discutimos las características clínicas, radiológicas y las alternativas terapéuticas de esta rara entidad.

\section{Caso clínico}

Un enfermo de 47 años de edad, con antecedentes de hipertensión arterial, fue encontrado inconsciente en la calle. Presentaba signos de traumatismo facial como consecuencia de la caída y fue trasladado a un hospital secundario. Presentó una crisis convulsiva generalizada de la que se recuperó poco después y se le practicó una tomografía computarizada (TC) cerebral. Con el diagnóstico de hemorragia subaracnoidea fue trasladado a nuestro hospital 5 horas más tarde. El paciente estaba consciente y orientado y refería intensa cefalea. Tenía rigidez de nuca y signos meníngeos positivos y no existía focalidad neurológica (Grado 1 de la clasificación de HSA de Hunt-Hess). La TC mostraba hemorragia subaracnoidea masiva en las cisternas de la base, rodeando al tronco cerebral y en la fosa posterior izquierda (Grado 3 de Fisher). También se podía apreciar un discreto aumento del tamaño ventricular (Fig. 1).

Al día siguiente se realizó una angiografía cerebral que evidenció una dilatación fusiforme en la porción anterobulbar de la PICA izquierda. La arteria vertebral era 


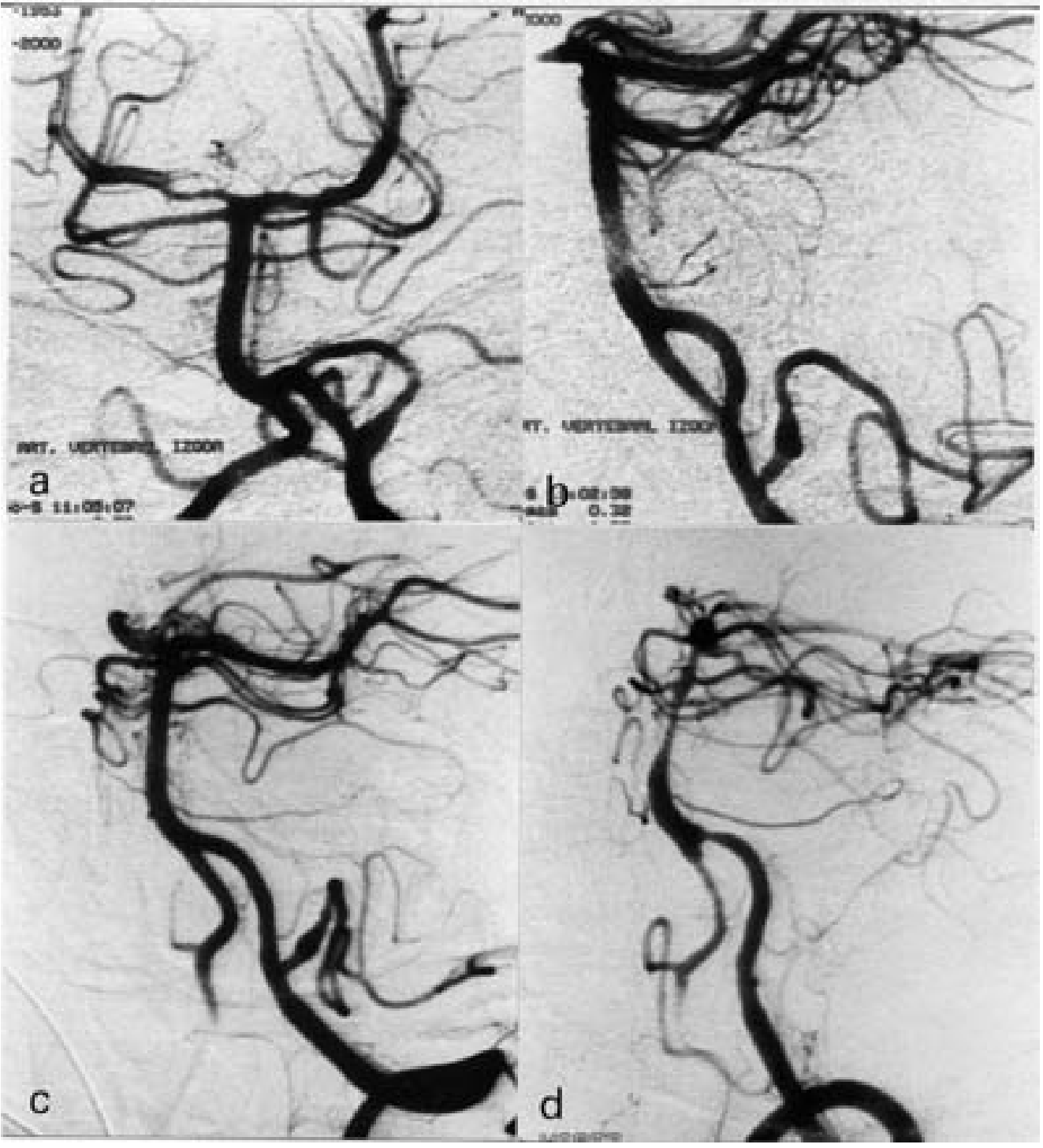

Figura 2. Estudio angiográfico; a) En la proyección anteroposterior se ve la dilatación fusiforme en la salida de arteria cerebelosa posteroinferior izquierda (PICA). Existe una duplicidad de las arterias cerebelosas superiores en el lado izquierdo y una salida algo distal de las arterias cerebelosas anteroinferiores; $\left.\begin{array}{llll}b & y & c\end{array}\right)$ Las proyecciones lateral $y$ oblicua muestran que el inicio de la PICA es normal y poco después la dilatación correspondiente al aneurisma. Un poco por encima de la PICA se ve que sale de la arteria vertebral una perforante. A nivel del tronco basilar se aprecia otra arteria perforante; d) Angiografia postquirúrgica que muestra la exclusión de la PICA y un vasoespasmo generalizado del territorio vertebrobasilar pero con las perforantes anteriormente mencionadas patentes.

normal así como el origen de la PICA. La arteria cerebelosa anteroinferior (AICA) tenía un origen más distal de lo habitual y se podían apreciar arterias perforantes procedentes tanto del tronco basilar como de la arteria vertebral distal a la PICA (Fig. 2). Con el diagnóstico de aneurisma disecante de la PICA el enfermo fue intervenido 11 días después de su ingreso. Se practicó una craniectomía suboccipital izquierda y se comprobó que el origen de la PICA era normal, presentando luego la arteria una dilatación irregular, de unos 7 milímetros de longitud, con una coloración negruzca y una pequeña burbuja hija. No se vieron arterias perforantes saliendo de este segmento anormal de la arteria ni de la porción laterobulbar, y se colocó un clip proximal y otro distal al aneurisma. El postoperatorio transcurrió sin complicaciones y el enfermo no presentó ningún déficit neurológico. Varios días después se realizó una angiografía de control que mostraba un importante vasoespasmo de todo el territorio vertebrobasilar y la exclusión del aneurisma y de la PICA. Un estudio de resonancia magnética (RM) previo al alta del enfermo descartó zonas de isquemia en tronco cerebral y cerebelo. Tres años después el paciente se encontraba asintomático.

\section{Discusión}

La incidencia de los aneurismas disecantes intracraneales no es conocida. Son más frecuentes en el territorio vertebrobasilar que en el carotídeo ${ }^{34}$. La forma de presentación dependerá del plano de la pared arterial en el que se localiza la disección. Así, si asienta entre la elástica interna y la media se producirá un hematoma subintimal con la consiguiente oclusión del vaso e isquemia. Si la disección es entre la capa media y la adventicia el resultado será de una $\mathrm{HSA}^{36}$. 
Los aneurismas disecantes que afectan únicamente a la PICA son muy raros. En los casos publicados la edad osciló entre los 22 y 70 años (media 45,5), con una mayor incidencia en la $5^{\mathrm{a}}$ y $6^{\mathrm{a}}$ décadas. No existió clara distinción por sexo, con 19 hombres afectos y 16 mujeres. La etiopatogenia es desconocida y se han invocado numerosos factores predisponentes tales como displasia fibromuscular, poliquistosis renal, defectos congénitos y adquiridos de la pared vascular e hipertensión arterial, pero sin que ninguno haya sido probado. De los 35 casos publicados sólo 8 eran hipertensos como nuestro enfermo.

La presentación clínica más frecuente es como HSA. Así 26 enfermos presentaron síntomas y signos compatibles con HSA, que se demostró posteriormente en la TC, y 9 con grados variables de síndrome de Wallenberg (déficit sensitivo facial ipsilateral y de hemicuerpo contralateral, síndrome de Homer y ataxia) o infarto cerebeloso.

En el estudio angiográfico el aneurisma estaba localizado en las tres primeras porciones de la PICA (anterobulbar, laterobulbar y tonsilobulbar) en el $83 \%$ de los enfermos, y en el $50 \%$ de los casos en la porción más proximal, es decir la anterobulbar. En 25 casos se localizaba en el lado izquierdo y en 11 en el derecho, ya que existió un enfermo con aneurismas bilaterales. En la mayor parte de los casos el aneurisma adoptó una forma de dilatación fusiforme o irregular de la arteria, que se asoció frecuentemente a grados variables de estrechamiento del vaso (signo de "pearl and string"). En cualquier caso la mejor manera de demostrar el aneurisma disecante es la RM que permite apreciar el trombo dentro de la falsa luz del vaso ${ }^{11,20,23}$. Desgraciadamente en nuestro caso no fue posible realizar dicha exploración antes de la intervención.

La mayor parte de los autores están de acuerdo en que los enfermos que se presentan con HSA deben ser tratados ya sea con cirugía o con métodos endovasculares $^{2,7,19,27,37}$. Más dudas plantean los que producen isquemia ya que existe la posibilidad de que la disección progrese. De los 35 casos publicados únicamente 3 se trataron de forma conservadora. Dos tenían un síndrome isquémico $y$ otro fue el enfermo con aneurisma bilateral. Este inicialmente presentó isquemia y luego HSA falleciendo tras ésta de forma fulminante.

El tratamiento de estos aneurismas es controvertido, dadas las características de la PICA, ya que de sus tres primeras porciones surgen perforantes que irrigan la parte lateral del bulbo y en un tercio de los casos la vascularización depende exclusivamente de ella. Se han realizado múltiples tipos de tratamientoquirúrgico, talescomoreconstruccióndel aneurisma con clips especiales y recubrimiento del mismo (5 casos), colocación de clip proximal (1 caso), colocación de clip proximal y distal con o sin resección del aneurisma (10 casos), y en 6 casos además de ésto se procedió a practicar una revascularización mediante anastomosis de la PICA distal a la arteria occipital. En 10 enfermos se utilizó un procedimiento endovascular para excluir el aneurisma. Recientemente se ha publicado una serie de 6 pacientes tratados con oclusión endovascular del aneurisma tras comprobar, en el estudio angiográfico, una aceptable circulación de suplencia, que dependía del tamaño de la AICA. En 2 casos en que la AICA era de un calibre menor de la mitad de la PICA realizaron previamente al tratamiento un test de oclusión ${ }^{17}$.

Nosotros pensamos, al igual que otros autores ${ }^{3}$, que la exploración quirúrgica es superior al tratamiento endovascular ya que permite una visión directa del segmento arterial afecto y comprobar si salen de él o no ramas perforantes y según el caso realizar el tratamiento más adecuado. Si no existen perforantes como en nuestro enfermo se puede sacrificar esa parte del vaso. La pertinencia de realizar además un "by-pass" de la PICA distal al aneurisma, que es sin duda un procedimiento muy elegante, es recomendado por varios autores ${ }^{8,10-12,20,32}$. Sin embargo, para otros es discutible, ya que únicamente permitiría revascularizar las partes más distales de la PICA y no el segmento de la arteria enfermo del que se originan las perforantes ${ }^{21,25}$.

De los 32 enfermos tratados con cualquier tipo de procedimiento el resultado fue bueno en 26. Cuatro pacientes tuvieron algún tipo de déficit neurológico, pero en sólo uno fue definitivo. Dos enfermos fallecieron, uno de ellos por resangrado tras tratamiento quirúrgico con recubrimiento con músculo del aneurisma (técnica hoy día considerada como insuficiente) y otro que fue intervenido en fase aguda y con muy mal grado clínico. Podemos pues concluir que tanto las técnicas quirúrgicas como las endovasculares son satisfactorias en el tratamiento de estos pacientes.

\section{Bibliografía}

1. Ali, M.J., Bendok, B.R., Tawk, R.G., Getch, C.C., Batjer, H.H.: Trapping and revascularization for a dissecting aneurysm of the proximal posteroinferior cerebellar artery: Technical case report and review of the literature. Neurosurgery 2002; 51: 258-263.

2. Aoki, N., Sakai, T.: Rebleeding from intracranial dissecting aneurysm in the vertebral artery. Stroke 1990; 21: 16281631.

3. Day. A.L., Cawley, C.M.: A dissecting aneurysm of the posteroinferior cerebellar artery: Case report. Neurosurgery 1998; 43: 356 (Comment).

4. Dinichert, A., Rüfenacht, D.A., de Tribolet, N.: Dissecting aneurysms of the posterior inferior cerebellar artery: report of four cases and review of the literature. J Clin Neurosci 2000; 7: 515-520.

5. Fransen, P., de Tribolet, N.: Dissecting aneurysm of the posterior inferior cerebellar artery. Br J Neurosurg 1994; 8: 381-386. 
6. Friedman, A.H., Drake, C.G.: Subarachnoid hemorrhage from intracranial dissecting aneurysm. J. Neurosurg 1984; 60: 325-334.

7. Gómez, P.A., Campollo, J., Lobato, R.D., Lagares, A., Alén, J.F.: Hemorragia subaracnoidea secundaria a aneurismas disecantes de la arteria vertebral. Descripción de 2 casos y revisión de la literatura. Neurocirugía 2001; 12 : 499-508.

8. Hashimoto, T., Kanki, T., Abe, S., Nakazawa, K., Nakamura, N.: Dissecting aneurysm of the vertebro-basilar system: surgical treatment in cases with brainstem isquemia. Jpn J Stroke 1992; 14: 355-360 (Jpn).

9. Hudgins, R.J., Day, A.L., Quisling, R.G., Rhoton, Jr. A.L., Sypert, G.W., García-Bengochea, F.: Aneurysms of the posterior inferior cerebellar artery. A clinical and anatomical analysis. J. Neurosurg 1983; 58: 381-387.

10. Jafar, J.J., Kamiryo, T., Chiles, B.W., Nelson, P.K.: A dissecting aneurysm of the posterior cerebellar artery : Case report. Neurosurgery 1998; 43: 353-356.

11. Kanou, Y., Arita, K., Kurisu, K., Ikawa, F., Eguchi, K., Monden, S., et al.: Dissecting aneurysm of the peripheral posterior inferior cerebellar artery. Acta Neurochir (Wien) 2000; 142: 1151-1156.

12. Kawaguchi, S., Sakaki, T., Kamada, K., Iwanaga, H., Takehashi, K., Tsujimoto, M.: Dissecting aneurysm of the posterior inferior cerebellar artery. Case report. Neurol Med Chir (Tokio) 1993; 33: 634-637 (Jpn).

13. Komiya, H., Saeki, N., Iwadate, Y., Sunami, K., Yamaura, A.: Posterior inferior cerebellar artery dissecting aneurysm presenting with Wallenberg's syndrome. Case report. Neurol Med Chir (Tokio) 1988; 28: 404-408 (Jpn).

14. Kopera, M., Majchrzak, H., Ladzinski, P., Stech, W., Maliszewski, M.: Dissecting aneurysm of the posterior inferior cerebellar artery. Neurol Neurochir Pol 1992; 26: 897-901 (Polish).

15. Lefkowitz, M.A., Teitelbaum; G.P., Giannotta, S.L.: Endovascular treatment of a dissecting posterioinferior cerebellar artery aneurysm: Case report. Neurosurgery 1996; 39 : 1036-1039.

16. Locksley, H.B.: Report on the Cooperative Study of Intracranial Aneurysms and Subarachnoid Hemorrhage. Section V, Part I. Natural history of subarachnoid hemorrhage, intracranial aneurysms, and arteriovenous malformations. Based on 6368 cases in the Cooperative Study. J. Neurosurg 1966; 25: 219-239.

17. Maimon, S., Saraf-Lavi, E., Rappaport, Z.H., Bachar, G.: Endovascular treatment of isolated dissecting aneurysm of the posterior inferior cerebellar artery. AJNR 2006; 27: 527532 .

18. Mizushima, H., Sasaki, K., Kunii, N., Nishino, T., Jinbo, H., Abe, T., et al.: Dissecting aneurysm in the proximal region of the posterior inferior cerebellar artery presenting as Wallenberg's syndrome. Case report. Neurol Med Chir (Tokio)
2007; 18:

1994; 34: 307-310 (Jpn).

19. Mizutani, T., Aruga, T., Kirino, T., Miki, Y., Saito, I., Tsuchida, T.: Recurrent subarachnoid hemorrhage from untreated ruptured vertebrobasilar dissecting aneurysms. Neurosurgery $1995 ; 36$ : 905-913.

20. Nagahiro, S., Goto, S., Yoshioka, S., Ushio, Y.: Dissecting aneurysm of the posterior inferior cerebellar artery: Case report. Neurosurgery 1993; 33: 739-742.

21. Newell, D.W., Berger, M.S.: Dissecting aneurysm of the posterior inferior cerebellar artery: Case report. Neurosurgery 1993; 33: 742 (Comment).

22. Nishino, A., Sakurai, Y., Niizuma, H., Satoh, H., Kayama, T.: Dissecting aneurysm of distal posterior inferior cerebellar artery. Case report and review of the literature. No To Shinkei 1991; 43: 381-386 (Jpn).

23. Quint, D.J., Spickler, E.M.: Magnetic resonance demonstration of vertebral artery dissection. Report of two cases. J. Neurosurg 1990; 72: 964-967.

24. Shinoda, S., Murata, H., Waga, S., Kojima, T.: Bilateral spontaneous dissection of the posteroinferior cerebellar arteries: Case report. Neurosurgery 1998; 43: 357-359.

25. Solomon, R.A.: Trapping and revascularization for a dissecting aneurysm of the proximal posteroinferior cerebellar artery: Technical case report and review of the literature. Neurosurgery 2002; 51: 263 (Comment).

26. Takahashi, I., Takamura, H., Gotoh, S., Sasaki, H., Makino, K., Suzuki, N., et al.: Dissecting aneurysm of the posterior inferior cerebellar artery. A case report. No Shinkei Geka 1992; 20: 277-281 (Jpn).

27. Tawk, R.G., Bendok, B.R., Qureshi, Al, Getch, C.C., Srinivasan, J., Alberts, M., et al.: Isolated dissections and dissecting aneurysms of the posterior inferior cerebellar artery: topic and literature review. Neurosurg Rev 2003; 26: 180187.

28. Tikkakoski, T., Leinonen, S., Siniluoto, T., Koivukangas, J.: Isolated dissecting aneurysm of the left posterior inferior cerebellar artery: Endovascular treatment with a Guglielmi detachable coil. AJNR 1997; 18: 936-938.

29. Ueki, K., Teraoka, A., Yoshida, S., Hori, T.: Dissecting aneurysm of the posterior cerebellar artery. A case report. No Shinkei Geka 1987; 15: 1215-1219 (Jpn).

30. Wakamoto, H., Orii, M., Miyazaki, H., Ishiyama, N.: A dissecting aneurysm of the posterior inferior cerebellar artery was reduced spontaneously during conservative therapy: Case report. No Shinkei Geka 2002; 30: 425-429 (Jpn).

31. Wetjen, N.M., Link, M.J., Reimer, R., Nichols, D.A., Giannini, C.: Clinical presentation and surgical management of dissecting posterior inferior cerebellar artery aneurysms: 2 case reports. Surg Neurol 2005; 64: 462-467.

32. Yamakawa, H., Kaku, Y., Yoshimura, S., Ohkuma, A., Sakai, N.: Two cases of dissecting aneurysm of the distal posterior inferior cerebellar artery: possible involvement of 
segmental mediolytic arteriopathy in the pathogenesis. Clin Neurol Neurosurg 2005; 107: 117-122.

33. Yamaura, A., Isobe, K., Karasudani, H., Tanaka, M., Komiya, H.: Dissecting aneurysms of the posterior inferior cerebellar artery. Neurosurgery 1991; 28: 894-898.

34. Yamaura, A., Ono, J., Hirai, S.: Clinical picture of intracranial non-traumatic dissecting aneurysm. Neuropathology 2000; 20: 85-90.

35. Yamaura, I., Tani, E., Yolota, M., Nakano, A., Fukami, M., Kaba, K., et al.: Endovascular treatment of ruptured dissecting aneurysms aimed at occlusion of the dissected site by using Guglielmi detachable coils. J. Neurosurg 1999; 90: 853856.

36. Yonas, H., Agamanolis, D., Takaoka, Y., White, R.J.:
Dissecting intracranial aneurysms. Surg Neurol 1977; 8: 407415.

37. Yuki, I., Murayama, Y., Viñuela, F.: Endovascular management of dissecting vertebrobasilar artery aneurysms in patients presenting with acute subarachnoid hemorrhage. J. Neurosurg 2005; 103: 649-655.

Rivas, J.J.; Domínguez, J.; Bravo, P.; Pérez, J.; Avila, A.P.: Aneurisma disecante de la arteria cerebelosa posteroinferior. Neurocirugía 2007; 18: 232-237.

Correspondencia postal: Juan José Rivas Salas. Servicio de Neurocirugía. Hospital 12 de Octubre. Avenida de Córdoba s/n. 28041 Madrid 